\title{
Modelos Recursivos de Índice de Masa Corporal con Variables Sociodemográficas, Funciones del Yo y Alteración Alimentaria en Mujeres Mexicanas
}

\author{
Recursive Models of Body Mass Index With \\ Sociodemographic Variables, Ego Functions, \\ and Eating Disorders in Mexican Women
}

\author{
Cecilia Meza y José Moral \\ Universidad Autónoma de Nuevo León
}

\begin{abstract}
El objetivo del estudio fue generar modelos recursivos para predecir el índice de masa corporal (IMC) en mujeres mexicanas, incluyendo variables sociodemográficas, funciones del yo y aspectos de alteración alimentaria. El Cuestionario para la Evaluación de las Funciones del Yo (EFA; Bellak \& Goldsmith, 1993) y el Cuestionario de Sobreingesta Alimentaria (OQ; O'Donnell \& Warren, 2007) se aplicaron a una muestra por cuotas del IMC de 217 mujeres de 20 a 40 años de Nuevo León. Se definieron 3 modelos con buen ajuste a los datos. En el modelo más complejo mayor IMC es pronosticado por menor nivel socioeconómico, mayor edad y comer excesivo. Mayor IMC es predictor de motivación para bajar de peso e insatisfacción con la imagen corporal. La insatisfacción con la imagen corporal predice motivación para bajar de peso. Peores hábitos de salud pronostican menor motivación para bajar de peso, mayor insatisfacción con la imagen corporal y comer excesivo. La alteración afectiva predice insatisfacción con la imagen corporal y comer excesivo. En base a estos modelos se formulan sugerencias para comprender el sobrepeso y la obesidad.
\end{abstract}

Palabras clave: obesidad, sobrepeso, funciones del yo, sobreingesta, imagen corporal

\begin{abstract}
The objective of the study was to generate recursive models to predict the body mass index (BMI) in Mexican women, including socio-demographic variables, ego functions and eating alteration aspects. The Ego Functions Assessment (EFA; Bellak \& Goldsmith, 1993) and the Overeating Questionnaire (OQ; O'Donnell \& Warren, 2007) were applied to a quota sample of the BMI of 217 women aged 2040 residing in Nuevo León. Three models with a good fit to data were defined. In the most complex model higher BMI is predicted by lower socioeconomic status, older age and excessive eating. A higher BMI is a predictor of motivation to lose weight and body image dissatisfaction. Dissatisfaction with body image predicts motivation to lose weight. Worse health habits predict lower motivation to lose weight, greater body dissatisfaction, and excessive eating. Affective disorder predicts body image dissatisfaction and excessive eating. Based on these models, suggestions are formulated to understand overweight and obesity.
\end{abstract}

Keywords: obesity, overweight, ego functions, overeating, body image

\section{Datos Epidemiológicos de la Obesidad en México}

El sobrepeso y la obesidad son un problema de salud que contribuyen a muchas otras enfermedades, como diabetes mellitus, cardiopatías, cáncer y reumatismo (Himmelgreen, 2007; Montero, 2001), las cuales generan altos costos en atención hospitalaria (Taylor, 2007). La pérdida de productividad por muertes prematuras atribuibles a la obesidad y al sobrepeso presenta una tasa de crecimiento promedio anual del 13,5\% (México, Secretaría de Salud, 2010).

Cecilia Meza Peña y José Moral de la Rubia, Facultad de Psicología, Universidad Autónoma de Nuevo León, Monterrey, México.

La correspondencia relativa a este artículo debe ser dirigida a la Dra. Cecilia Meza Peña, Facultad de Psicología, Universidad Autónoma de Nuevo León, Dr. Carlos Canseco 110, Col. Mitras Centro, 64460, Monterrey, Nuevo León, México. E-mail: cecilia.meza@gmail.com 
Los resultados de la Encuesta Nacional de Salud y Nutrición 2006 (Olaiz et al., 2006) establecen que la obesidad y el sobrepeso están presentes en el 70\% de la población mexicana adulta de ambos sexos, siendo mayor el porcentaje en mujeres (72\%) que en hombres (67\%). México ocupa el segundo lugar en obesidad en relación a una evaluación de salud entre 30 países. Las tasas de obesidad de mexicanos solo son superadas por los Estados Unidos (Organization for Economic Co-operation and Development, 2008).

La prevalencia de la obesidad no es igual en los diferentes estratos socioeconómicos ni por género (Crosnoe, 2007; Flores, Carrión \& Barquera, 2005; Hernández et al., 1996). El sobrepeso y obesidad afectan más a las mujeres adultas que a los hombres, aumentando el riesgo después de los 20 años para ambos sexos (Hernández et al., 1996; México, Instituto Nacional de Salud Pública, 2007). Otros estudios señalan que el sobrepeso de la madre está asociado de forma directa con el sobrepeso del hijo (Flores et al., 2005; Junien \& Nathanielsz, 2007). Además, factores socioeconómicos, como el desempleo y el bajo nivel educativo, tienen un alto impacto en el desarrollo de la obesidad (Kilicarslan et al., 2006; Renman, Engström, Silfverdal \& Åman, 1999).

\section{El Enfoque Biopsicosocial de la Obesidad}

La literatura científica ofrece un amplio número de teorías, desde biológicas, sociales y psicológicas, así como perspectivas explicativas para el problema de la obesidad, como la cognitiva, conductual, sistémica y de adicciones. No obstante, ninguna es suficiente por sí misma para explicar el problema (Chinchilla, 2005; Stroe, 1995; Taylor, 2007). Algunos autores advierten que la génesis de la obesidad es compleja, ya que no solo se trata de sobreingesta, sino que en ella confluyen eventos de vida estresantes, además de la utilización de la comida como refugio para compensar frustraciones, tristeza, necesidades y temores (Cabello \& Zúñiga, 2007).

En un intento integrador de las diversas perspectivas que contemplan los intereses de las tres principales teorías, surge lo que se conoce como enfoque biopsicosocial, ofreciendo una imagen holística de la conducta alimentaria, en el que se reconoce que esta conducta está mediada por variables fisiológicas, psicológicas y sociales, ya que la obesidad puede ser una condición crónica en la cual confluyen muchos factores, como hereditarios, nutricionales, de gasto energético, socioeconómicos y psicológicos (Stunkard, 2000; Taylor, 2007).

\section{La Dimensión Psicológica de la Obesidad}

En la dimensión psicológica de la obesidad se ha documentado la presencia en mujeres obesas de alteraciones en la percepción de la imagen corporal (Sansone, Wiederman \& Monteith, 2001), síntomas depresivos en relación con el tamaño del cuerpo (Lim, Thomas, Bardwell \& Dimsdale, 2008), bajo control de impulsos y en particular hacia la comida (Rand \& Stunkard, 1978), mayor presencia de insatisfacción corporal, perfeccionismo, obsesión por el peso, figura y comida, así como baja autoestima (Arias et al., 2006) y dificultades para el manejo de la agresividad y la asertividad (Hidalgo \& Gottlieb, 2001). Todos estos son factores de riesgo evaluados por el Cuestionario de Sobreingesta (OQ; O’Donnell \& Warren, 2007).

Desde una perspectiva psicoanalítica, McCall (1974) y Wadden y Lucas (1980) analizan la fortaleza del yo de pacientes obesos en distintos escenarios clínicos, estableciendo que el fortalecimiento del yo lleva a un sentido de control que posibilita la pérdida de peso y, por tanto, la fortaleza del yo se puede utilizar como predictor del éxito en estos tratamientos. Aspectos del yo, como el control de impulsos, el sentido de realidad, el funcionamiento defensivo y las relaciones objetales, se resaltan como relevantes para el éxito del tratamiento de la obesidad (Bruch, 1973; Stunkard, 1959). 
Adoptando una perspectiva psicológica, el estudio que se reporta tuvo como objetivo determinar las variables sociodemográficas (edad, escolaridad, estatus socioeconómico y número de hijos), funciones del yo (sentido de realidad, relaciones objetales, regulación de impulsos y funcionamiento defensivo), aspectos cognitivos (imagen corporal, motivación para bajar de peso, sentimientos hacia la comida), aspectos afectivos (alteración) y aspectos sociales (aislamiento) que están relacionados y permiten predecir el índice de masa corporal (IMC) en mujeres mexicanas. Los determinantes biológicos (hormonas y genética) y sociales (representaciones y prácticas sociales) no se incluyeron a fin de profundizar en lo psicológico.

Se esperaba que las mujeres de mayor edad, menor nivel socioeconómico (NSE) y de escolaridad y mayor número de hijos presentaran un mayor IMC, como refleja la encuesta nacional mexicana (Olaiz et al., 2006). Así, las mujeres solteras frente a las casadas presentarían un menor IMC, al ser usualmente más jóvenes y no tener hijos. En relación con las funciones del yo, se esperaba encontrar un mayor IMC mientras peor fuera la regulación de afectos, instintos e impulsos, más problemas de relaciones objetales hubiera, mayor fuera el funcionamiento defensivo y peor el sentido de la realidad. En relación con los factores del cuestionario OQ, se esperaba encontrar un mayor IMC a mayor sobreingesta, mayor frecuencia de conducta de antojos alimentarios, mayor insatisfacción con la imagen corporal, mayor motivación para bajar de peso y mayor alteración afectiva y aislamiento social.

\section{Método}

\section{Participantes}

Se empleó un muestreo no probabilístico por cuotas de grupos de IMC: 50\% sobrepeso y obesidad y 50\% normopeso. Para la recolección de los datos se implementaron dos estrategias: (a) invitación a mujeres en una clínica de salud privada para control de peso $(n=60)$ y (b) invitación a la Facultad de Psicología de una universidad de Nuevo León en México, a través de los alumnos de licenciatura $(n=157)$.

Como criterios de inclusión se estableció que todas las participantes fueran mujeres residentes de la Ciudad de Monterrey, de 20 a 40 años cumplidos, que hubieran aceptado la participación en el estudio y cuyo IMC cayera en rangos de normopeso hasta obesidad para completar las siguientes cuotas: $50 \%$ normopeso, $25 \%$ sobrepeso y $25 \%$ obesidad. Como criterios de exclusión se establecieron los siguientes: mujeres embarazadas y aquellas personas que rechazaron participar en el estudio o que dejaron incompletos los protocolos.

El total de participantes, todas mujeres de Monterrey, Nuevo León, México, fue de 217. De ellas, el 49,3\% (107) estaba en el rango de normopeso ( $M$ de $\mathrm{IMC}=21,42, D E=2,36)$, el $25,3 \%$ (55), en el rango de sobrepeso $(M$ de IMC $=27,66, D E=1,48)$ y el $25,3 \%(55)$, en el rango de obesidad $(M$ de IMC $=34,99, D E=4,45)$. Dentro del grupo de mujeres obesas, el 60\% (33) presentaba obesidad leve (IMC de 30 a 34,9), 31\% (17), moderada (IMC de 35 a 39,9) y 9\% (5), mórbida (IMC de 40 o mayor). Para definir estos grupos se emplearon los rangos de IMC definidos por la World Health Organization (2000).

Las edades oscilaron entre los 20 y 40 años, con una media de 26 años y una desviación estándar de 6,8 años. El 1,5\% de las participantes tenía estudios de primaria, 5,5\% de secundaria, 44\% de media superior y $49 \%$ universitarios. El $69 \%$ de la muestra dijo pertenecer a clase media, $18 \%$ a media alta, $11 \%$ a media baja, $1 \%$ a baja y otro $1 \%$ a alta. El $71 \%$ de las participantes eran solteras, $26 \%$ casadas, $2 \%$ divorciadas o separadas y 1\% vivía en unión libre. El 74\% de las mujeres encuestadas no tenía hijos y, entre las mujeres que sí reportaron tener hijos, el valor modal fue de 2 hijos. 


\section{Instrumentos}

Cuestionario Sociodemográfico. Se formularon ocho preguntas para obtener la información sociodemográfica: 1) domicilio; 2) colonia; 3) edad en años; 4) último año de educación completado (primaria, secundaria, preparatoria, licenciatura, maestría u otro); 5) clase social (nivel socioeconómico) al que considera que pertenece (baja, media-baja, media, media-alta y alta); 6) estado civil (soltera, casada, divorciada, viuda, separada, unión libre u otro); 7) tiene hijos (no o sí) y cuántos; 8) está embarazada (no o sí) y si lo está, semanas de gestación.

Cuestionario Para la Evaluación de las Funciones del Yo (EFA; Bellak \& Goldsmith, 1993). Evalúa el grado de ajuste y funcionamiento adaptativo en relación con 12 funciones del yo. Solo se incluyeron cuatro de las 12 funciones del yo evaluadas: (a) sentido de realidad, del mundo y de sí mismo; (b) regulación y control de instintos, afectos e impulsos; (c) relaciones objetales y (d) funcionamiento defensivo. Se incluyeron estas porque se consideraron relevantes para la obesidad; de ahí que se denominó Cuestionario para la Evaluación de las Funciones del Yo Críticas para la Obesidad. Cada función cuenta con 10 ítems, que se responden en forma autoaplicada en una escala tipo Likert, que varía de 0 (nunca) a 4 (siempre) puntos. El original tiene un formato de entrevista clínica, por lo que aquí se trata de una adaptación para que el encuestado respondiera por escrito sin requerir un entrevistador. Las puntuaciones altas reflejan un grado de funcionamiento pobre.

En la presente muestra los valores de ajuste por análisis factorial confirmatorio en cada escala oscilaron entre adecuados a buenos para modelos unidimensionales, excluyendo dos ítems en relaciones objetales y dos en funcionamiento defensivo. Excluidos estos cuatro ítems, los valores de consistencia interna, evaluada por alfa de Cronbach, variaron de $\alpha=0,65$ para regulación y control de instintos, afectos e impulsos a 0,75 para funcionamiento defensivo, con una media de 0,70 , considerando 36 ítems. Al considerar 32 ítems, un modelo de cuatro factores correlacionados, excluyendo dos ítems por factor, mostró un ajuste adecuado, $\chi^{2}(453, N=217)$ $=516,30, p=0,02, \chi^{2} / g l=1,14, \mathrm{RMSEA}=0,02, \mathrm{GFI}=0,85$ y AGFI $=0,83$.

Cuestionario de Sobreingesta Alimentaria (OQ; O’Donnell \& Warren, 2007). Evalúa factores de riesgo y protección asociados con el sobrepeso y la obesidad. Lo integran 11 escalas definidas a partir de análisis factorial exploratorio: Defensividad, Sobreingesta, Subingesta, Antojos alimentarios, Racionalizaciones, Motivación para bajar de peso, Hábitos de salud, Imagen corporal, Aislamiento social, Alteración afectiva y Expectativas relacionadas con el comer. Se compone de 80 ítems tipo Likert con un rango de respuesta de 5 puntos (de 0 para nada a 4 muchísimo). Los valores de consistencia interna de sus 11 factores varían de 0,79 a 0,88 , con un valor mediano de 0,82 , en una muestra de 1788 participantes estadounidenses de ambos sexos (O’Donnell \& Warren, 2007).

En el estudio que se reporta en este artículo se eliminaron 16 ítems, producto de los análisis factoriales (exploratorio y confirmatorio) y estimaciones de consistencia interna de los factores. De estos 16 ítems, ocho corresponden al factor Racionalizaciones, el cual desaparece, reduciéndose la escala a 64 ítems y 10 factores correlacionados. Todos los ítems se valoran en el sentido de alteración alimentaria, por lo que se invierte la puntuación de los seis ítems de Hábitos de salud, seis de Defensividad, seis de Imagen corporal y cuatro de Sentimientos positivos al comer nuevo factor encontrado en este estudio. Los valores de consistencia interna de los 10 factores, medida por alfa de Cronbach, variaron de 0,65 (Subingesta) a 0,87 (Imagen corporal y Motivación para bajar de peso), con una media de 0,80. La consistencia interna de los 64 ítems seleccionados es alta $(\alpha=0,85)$. Con estos 10 factores se obtuvieron índices de ajuste buenos: $\chi^{2} / g l=1,11$, RMSEA $=0,02$ y PNCP $=0,96$. Los ítems de los factores originales Expectativas relacionadas con el comer y Sobreingesta conforman dos nuevos factores, que 
son Comer excesivo y Sentimientos positivos al comer. Se construyó un índice de riesgo con la suma de las 10 escalas, el cual tiene una distribución normal. Un mayor puntaje refleja mayor sobreingesta alimentaria. Para mayores detalles ver Meza y Moral (en prensa).

\section{Procedimiento}

En el presente estudio se tomaron en cuenta las recomendaciones hechas por la American Psychological Association (APA) sobre los aspectos éticos para la investigación con seres humanos (APA, 2002) y de la Sociedad Mexicana de Psicología (2007). En virtud de ello, se dio a los participantes la información referente a los propósitos del estudio, se garantizó la confidencialidad de la información aportada y se solicitó su consentimiento firmado.

Las mediciones antropométricas y la aplicación de los instrumentos se realizaron en las instalaciones de la universidad en Nuevo León. La muestra fue levantada entre enero y septiembre de 2010.

La recolección de los datos se empezaba con el registro por el encuestador de los datos sociodemográficos y antropométricos. Para la medición de talla y peso se empleó una báscula clínica con estadímetro, la cual permite medir estatura hasta 1,95 metros, así como hacer lectura directa de peso en dos varas, una de $140 \mathrm{~kg}$ y otra de $20 \mathrm{~kg}$. Se proseguía con la administración individual del cuestionario autoaplicado, el que incluía el EFA adaptado y el OQ.

\section{Análisis de Datos}

Dado que en la encuesta ENSANUT 2006 (Olaiz et al., 2007) se maneja la obesidad y el sobrepeso como un grupo ( $M$ de IMC $=31,33, D E=4,94)$, este se compara con el normopeso ( $M$ de $\mathrm{IMC}=21,42, D E=2,36)$, dicotomizando así la variable continua de IMC.

Se estimaron correlaciones producto-momento de Pearson entre IMC y las variables sociodemográficas, funciones del yo y sobreingesta. Con los correlatos significativos se estimó un modelo de regresión lineal múltiple, empleando el método stepwise. Finalmente, se estimaron modelos de senderos, esto es, de variables latentes. Estos se estimaron por medio del método de Mínimos Cuadrados Generalizados (GLS).

Se contemplaron cinco índices de ajuste: $\chi^{2}$, cociente entre $\chi^{2}$ y sus grados de libertad $\left(\chi^{2} / g l\right)$, error cuadrático medio de aproximación (RMSEA) de Steiger-Lind, índice normado de Bentler-Bonett (NFI) e índice comparativo de Bentler-Bonett (CFI). Se estipularon como valores de buen ajuste para los índices: $p>0,05$ para $\chi^{2}$, cociente $\chi^{2} / g l<2$, RMSEA $\leq 0,05, \mathrm{NFI} \geq 0,90$ y CFI $\geq 0,95$; y como adecuado ajuste: $p>0,01$ para $\chi^{2}$, cociente $\chi^{2} / g l<3$, RMSEA $\leq 0,07, \mathrm{NFI} \geq 0,80$ y CFI $\geq 0,85$ (Moral, 2006).

\section{Resultados}

\section{Correlaciones del IMC con las Variables Independientes}

Respecto de las variables sociodemográficas, el IMC se correlacionó directamente con la edad, el número de hijos y el nivel de escolaridad e inversamente con el NSE; respecto de las funciones del yo, se correlacionó directamente solo con sentido de realidad (a mayor valor menor sentido de realidad); y respecto del cuestionario de sobreingesta, se correlacionó directamente con el puntaje total y con los factores Motivación para bajar de peso, Comer excesivo y Alteración afectiva e inversamente con los factores Imagen corporal y Hábitos de salud (véase la Tabla 1). 
Tabla 1

Correlaciones de las Variables Sociodemográficas, Funciones del Yo y Puntaje Total y Factores del Cuestionario de Sobreingesta con IMC

\begin{tabular}{lrr}
\hline \multicolumn{1}{c}{ Dimensión, variable, factor } & \multicolumn{2}{c}{$\mathrm{IMC}$} \\
\cline { 2 - 3 } & \multicolumn{1}{c}{$r$} & \multicolumn{1}{c}{$p$} \\
\hline Variables sociodemográficas & & \\
Edad & 0,40 & $<0,01$ \\
Último año de educación completado & 0,15 & 0,03 \\
Nivel socioeconómico al que considera que pertenece & $-0,21$ & $<0,01$ \\
Número de hijos & 0,21 & $<0,01$ \\
Funciones del yo & & \\
Sentido de la realidad & & 0,03 \\
Regulación y control de instintos, afectos e impulsos & 0,15 & 0,17 \\
Relaciones objetales & 0,09 & 0,24 \\
Funcionamiento defensivo & 0,08 & 0,62 \\
Puntaje total y factores del Cuestionario de Sobreingesta & 0,03 & \\
Puntaje total & & \\
Alteración afectiva & 0,40 & $<0,01$ \\
Antojos alimentarios & 0,19 & $<0,01$ \\
Motivación para bajar de peso & $-0,06$ & 0,36 \\
Aislamiento social & 0,49 & $<0,01$ \\
Defensividad & 0,03 & 0,71 \\
Subingesta & 0,03 & 0,68 \\
Hábitos de salud & $-0,01$ & 0,93 \\
Sentimientos positivos al comer & $-0,16$ & 0,02 \\
Comer excesivo & $-0,00$ & 0,94 \\
Imagen corporal & 0,33 & $<0,01$ \\
\hline & $-0,46$ & $<0,01$ \\
\hline
\end{tabular}

Nota. $r=$ coeficiente de correlación producto-momento de Pearson; $p=$ probabilidad de la prueba $Z$ con la hipótesis nula $r=0$.

\section{Modelo de Regresión del IMC}

Al calcular el modelo de regresión del IMC con sus 11 correlatos significativos, ingresaron seis variables: motivación para bajar de peso $(\beta=0,38, p<0,01)$, edad $(\beta=0,42, p<0,01)$, número de hijos $(\beta=-0,21, p<0,01)$, imagen corporal $(\beta=-0,19, p<0,01)$, hábitos de salud $(\beta=$ $-0,12, p=0,04)$ y NSE $(\beta=-0,11, p=0,04)$. El modelo es significativo, $F(6,210)=27,33, p<0,01$. Los predictores presentan colinealidad, como indican los valores de tolerancia (Tol) e inflación de la varianza (VIF) alejados de la unidad, así como valores de correlación parcial ( $r_{\mathrm{p}}$ ) y semiparcial $\left(r_{\mathrm{sp}}\right)$ que descienden en comparación con las correlaciones originales. La distribución de los residuos se ajusta a una curva normal, $Z_{K-S}=1,29, p=0,07$, y se cumple el supuesto de independencia entre los residuos, como evidencia la ausencia de tendencia lineal en la Figura 1, la que representa los valores pronosticados estandarizados y los residuos estandarizados. 


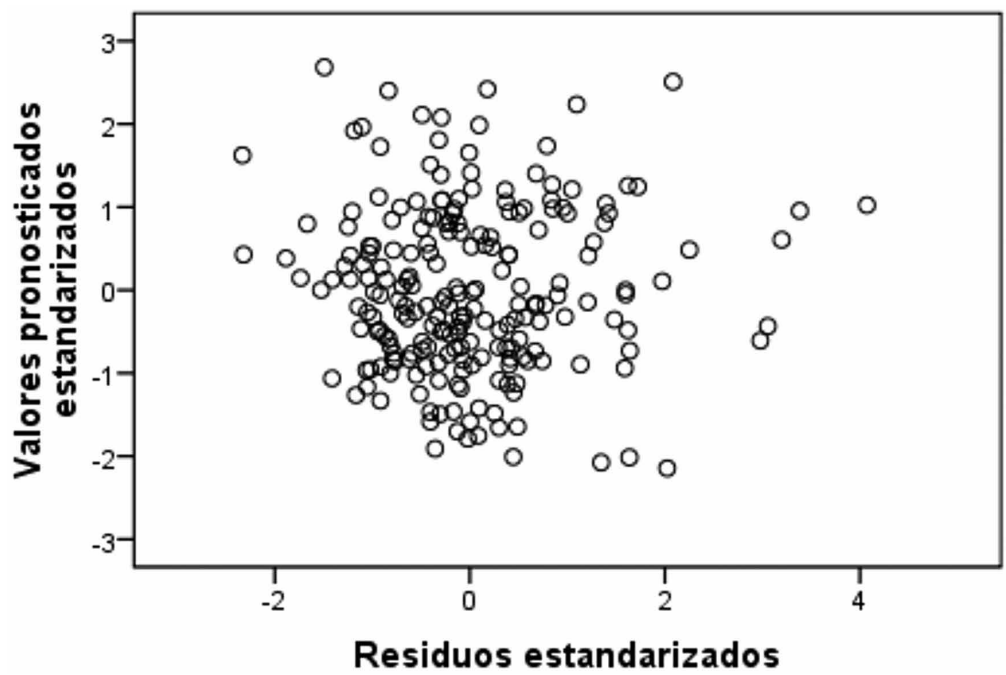

Figura 1. Diagrama de dispersión de los residuos estandarizados y los valores pronosticados estandarizados.

El modelo explica el $42 \%$ de la varianza del IMC $\left(R=0,66, R^{2}=0,44, R^{2} a j .=0,42\right)$, con un error estándar de estimación de 4,79. Mayor edad, mayor motivación para bajar de peso, menor número de hijos, mayor insatisfacción con la imagen corporal, peores hábitos de salud y menor NSE pronostican mayor IMC.

Debe señalarse que, si bien la correlación entre el número de hijos y el IMC es directa ( $r$ $=0,21$ ), el número de hijos es una variable con una fuerte colinealidad dentro del modelo de regresión ( $\mathrm{Tol}=0,48$ y FIV $=2,06)$, invirtiéndose el sentido de la relación por efecto de las otras variables, como lo refleja la correlación parcial $\left(r_{p}=-0,19\right)$ (véase la Tabla 2).

Tabla 2

Modelo de Regresión del Índice de Masa Corporal

\begin{tabular}{lrcccccccccc}
\hline \multirow{2}{*}{ Predictor } & \multicolumn{3}{c}{ Coeficientes } & \multicolumn{3}{c}{ Significación } & \multicolumn{3}{c}{ Correlaciones } & \multicolumn{3}{c}{ Colinealidad } \\
\cline { 2 - 11 } & \multicolumn{1}{c}{$B$} & $E E$ & $\beta$ & \multicolumn{1}{c}{$t$} & \multicolumn{1}{c}{$p$} & $r$ & $r_{\mathrm{p}}$ & $r_{\mathrm{sp}}$ & Tol & FIV \\
\hline Constante & 19,72 & 2,62 & & 7,54 & $<0,01$ & & & & & \\
Edad & 0,39 & 0,07 & 0,42 & 5,76 & $<0,01$ & 0,40 & 0,37 & 0,30 & 0,49 & 2,02 \\
MO & 0,30 & 0,05 & 0,38 & 6,26 & $<0,01$ & 0,49 & 0,40 & 0,32 & 0,73 & 1,37 \\
Número de hijos & $-1,36$ & 0,48 & $-0,21$ & $-2,84$ & $<0,01$ & 0,21 & $-0,19$ & $-0,15$ & 0,48 & 2,06 \\
CO & $-0,22$ & 0,07 & $-0,19$ & $-2,95$ & $<0,01$ & $-0,46$ & $-0,20$ & $-0,15$ & 0,66 & 1,51 \\
SA & $-0,17$ & 0,08 & $-0,12$ & $-2,02$ & 0,04 & $-0,16$ & $-0,14$ & $-0,10$ & 0,78 & 1,28 \\
NSE & $-1,14$ & 0,55 & $-0,11$ & $-2,08$ & 0,04 & $-0,21$ & $-0,14$ & $-0,11$ & 0,95 & 1,06 \\
\hline
\end{tabular}

Notas. Estimado por el método Stepwise. Predictores: MO = Motivación para bajar de peso; $\mathrm{CO}=$ Imagen corporal; $\mathrm{NSE}=$ Nivel socioeconómico y SA = Hábitos de salud. Coeficientes: $B=$ coeficiente de regresión sin estandarizar; $E E$ = error estándar de $B$ y $\beta=$ coeficiente de regresión estandarizado. Correlaciones de los predictores con el criterio (IMC): $r=$ coeficiente de correlación producto-momento de Pearson; $r_{\mathrm{p}}=$ correlación parcial y $r_{\mathrm{sp}}=$ correlación semiparcial. Colinealidad: Tol = tolerancia y FIV = factor de inflación de la varianza. Menor varianza compartida con los otros predictores (colinealidad) cuanto más próximos a 1 están ambos índices. 


\section{Modelos de Análisis de Senderos del IMC}

En primer lugar, se contrastó un modelo semejante al obtenido en la regresión múltiple. Se consideró la multicolinealidad entre edad, número de hijos, NSE y hábitos de salud. Se consideró el IMC como predictor de motivación para bajar de peso e imagen corporal, en el sentido que a mayor IMC hay mayor motivación para bajar de peso y peor imagen corporal. A su vez, se consideró la imagen corporal como predictor de motivación para bajar de peso, en el sentido que a peor imagen corporal mayor motivación para bajar de peso. Estas consideraciones se hicieron por tener estas vías un mayor sentido causal. Peores hábitos de salud pronostican menor motivación para bajar de peso y mayor insatisfacción con la imagen corporal.

El modelo explica el 20\% de la varianza del IMC, 37\% de la varianza de motivación para bajar de peso y $26 \%$ de la varianza de imagen corporal. Todos sus parámetros son significativos, con la excepción de la predicción del IMC por los hábitos de salud $(\beta=-0,09, p=0,13)$. El ajuste del modelo es bueno: $\chi^{2}(8, N=217)=10,93, p=0,21, \chi^{2} / g l=1,37$, NFI $=0,97$, CFI $=0,99$ y RMSEA $=0,04$.

En segundo lugar, si se elimina la vía de predicción del IMC por hábitos de salud (primera revisión del primer modelo), el porcentaje de varianza explicada del IMC es de $19 \%$ y el ajuste sigue siendo bueno, $\chi^{2}(9, N=217)=13,14, p=0,16, \chi^{2} / g l=1,46, \mathrm{NFI}=0,97, \mathrm{CFI}=0,99 \mathrm{y}$ RMSEA $=0,05$, pero la vía de predicción del IMC por el número de hijos solo se aproxima a la significación estadística $(\beta=-0,16, p=0,06)$, debido a su alta correlación con la edad $(r=0,71)$, adquiriendo mayor peso esta última en la predicción del IMC $(\beta=0,50, p<0,01)$.

Por último, si se elimina la vía de predicción del IMC por el número de hijos (segunda revisión del primer modelo), todos los parámetros resultan significativos. La varianza explicada del IMC es de 18\%, la de motivación para bajar de peso es $38 \%$ y la de la imagen corporal, $25 \%$. El ajuste del modelo es bueno: $\chi^{2}(6, N=217)=6,78, p=0,34, \chi^{2} / g l=1,13, \mathrm{NFI}=0,97$, CFI $=1$ y RMSEA = 0,02 (véase la Figura 2).

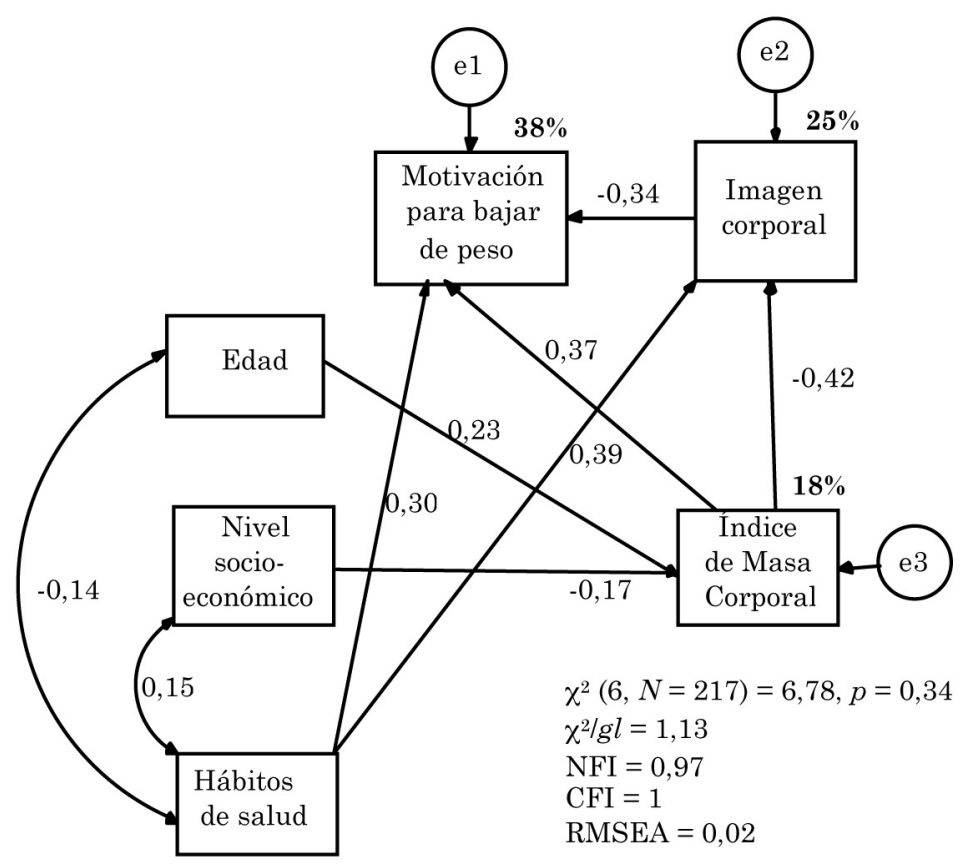

Figura 2. Parámetros estandarizados de la segunda revisión del primer modelo de IMC (con dos variables sociodemográficas y tres factores del OQ) estimado por GLS. 
Se estimó un segundo modelo en el que se introdujo una nueva variable manifiesta exógena, el comer excesivo, la cual es un predictor del IMC. El comer excesivo pronostica mayor IMC y se correlaciona directamente con la edad e inversamente con los hábitos de salud. En todo lo demás coincide con el primer modelo. El modelo explica el $26 \%$ de la varianza del IMC, $42 \%$ de motivación para bajar de peso y $28 \%$ de la insatisfacción con la imagen corporal. Todos sus parámetros son significativos. El ajuste a los datos es bueno: $\chi^{2}(7, N=217)=9,47, p=0,22, \chi^{2} /$ $g l=1,35, \mathrm{NFI}=0,97, \mathrm{CFI}=0,99$ y RMSEA = 0,04 (véase la Figura 3).

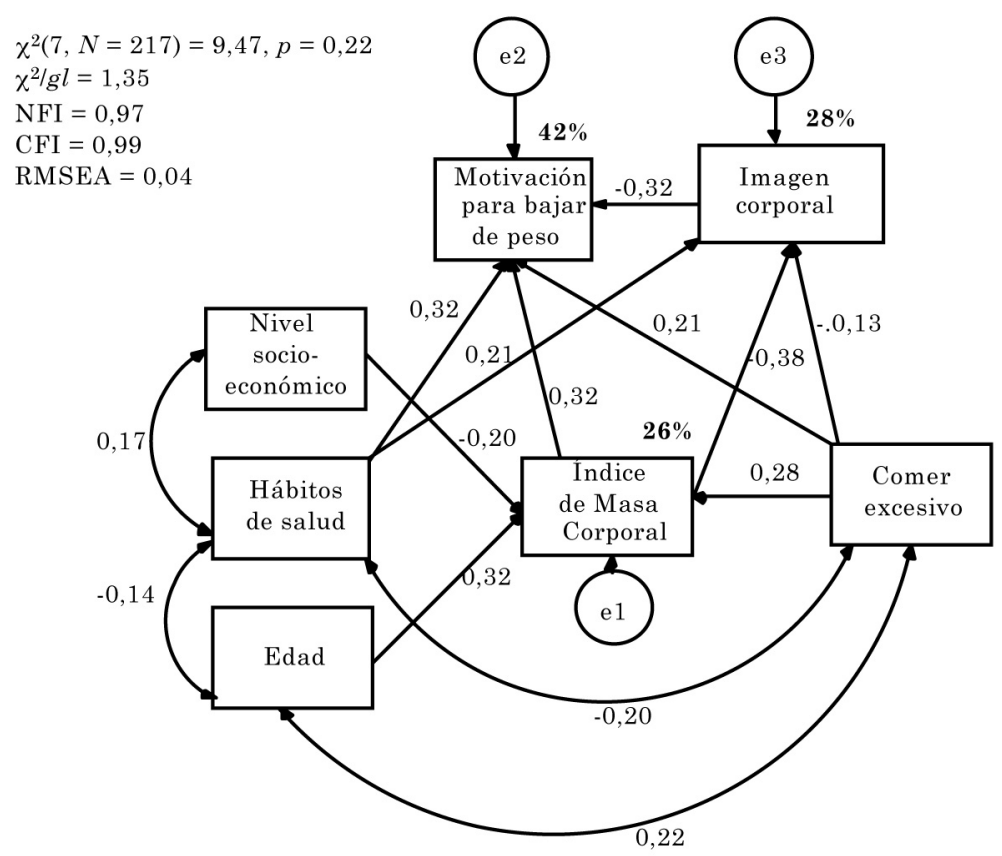

Figura 3. Parámetros estandarizados del segundo modelo de IMC (añadiendo el factor Comer excesivo de OQ) estimado por GLS.

En un tercer modelo se añadió la variable alteración afectiva, la que pronostica mayor insatisfacción con la imagen corporal y comer excesivo, además de correlacionarse con la edad. Esta vez se manejó el comer excesivo como una variable endógena, pronosticada por alteración afectiva y malos hábitos de salud, y no como variable exógena, como se hizo en el segundo modelo. El modelo explica el $24 \%$ de la varianza del IMC, $42 \%$ de la de motivación para bajar de peso, $32 \%$ de la de imagen corporal y $27 \%$ de la de comer excesivo. Todos sus parámetros son significativos, El ajuste a los datos por GLS es bueno: $\chi^{2}(13, N=217)=20,08, p=0,08, \chi^{2} / g l=$ $1,54, \mathrm{NFI}=0,95, \mathrm{CFI}=0,98$ y RMSEA $=0,05$. Con respecto al modelo anterior, el comer excesivo es una variable explicada, en la que la edad es un determinante no significativo, por lo que no se contempla esta vía. Además, refleja determinantes emocionales en el comer excesivo y la insatisfacción con la imagen corporal (véase la Figura 4). 


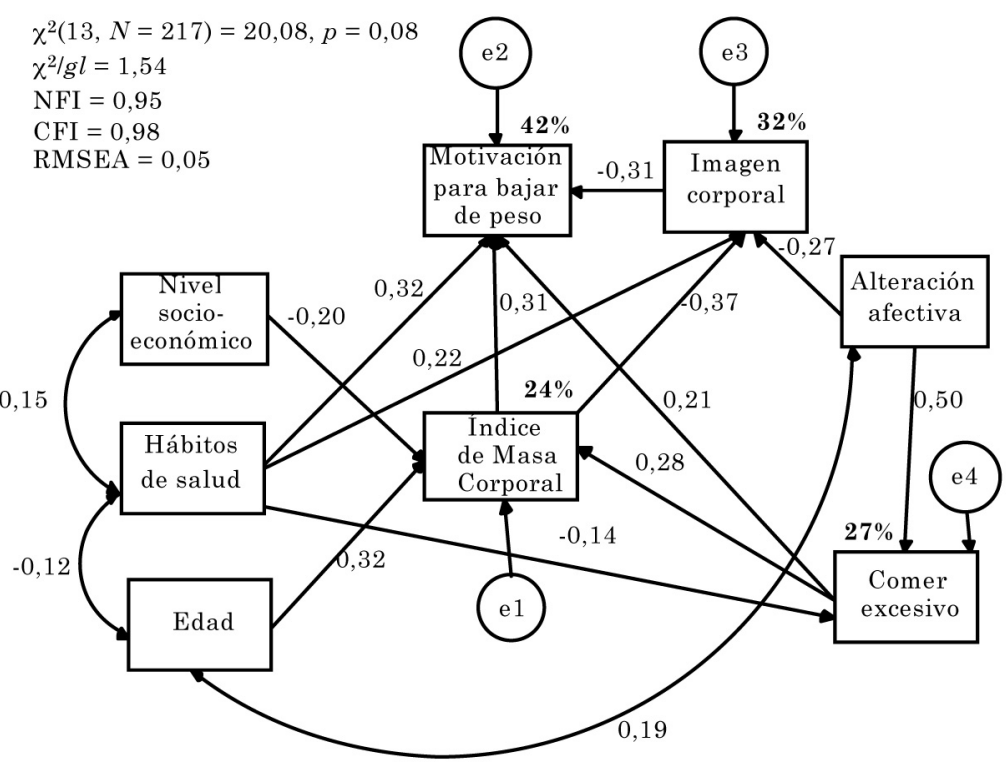

Figura 4. Parámetros estandarizados del tercer modelo de IMC (añadiendo además el factor Alteración afectiva del OQ) por GLS.

\section{Discusión}

Los resultados de este estudio dan cuenta de la relación que existe entre la obesidad y factores asociados a las conductas de sobreingesta alimentaria, en los que se incluye la conducta de comer en exceso y la pobreza en hábitos de salud, mismos factores que constituyen una causa clara de la obesidad. Además, se pudo observar que en el grupo de personas con obesidad existe una insatisfacción con su imagen corporal, carencia de sentido de la realidad, así como alteración afectiva, entendida como la presencia de aspectos depresivos y ansiógenos, de acuerdo a las variables evaluadas por este factor.

Dentro de estos hallazgos, resulta interesante dar cuenta de la presencia de alteraciones afectivas, en el sentido de conductas ansiógenas y depresivas que pueden estar impactando en los esfuerzos por controlar la dieta, así como en la imagen que la persona tiene de sí misma, afectando su sentido de realidad y su satisfacción con la imagen corporal. De este modo, sería comprensible que en las personas con sobrepeso/obesidad, aun en la presencia de una alta motivación para bajar de peso, esta presencia de conductas ansiógenas y depresivas puedan ser las que las llevan a comer en exceso, causando mayores frustraciones afectivas, por el bajo control de impulsos, y dañando el concepto de sí mismo.

Es así que en personas con obesidad se tendrían que proponer plataformas para trabajar la pobreza de hábitos de salud, buscando mejoras y cambios en los patrones de los mismos, analizando factores conductuales. Por ende, se tendría que trabajar el control de impulsos para evitar el comer en exceso, además de abordar aspectos emocionales. Las emociones de ansiedad y depresión pueden estar presentes por la dieta, insatisfacción con la imagen corporal o como factor de co-morbilidad en estos grupos poblacionales. Es posible que, en un intento por acallar estas emociones, se coma en exceso, dado que en la población mexicana se dice comúnmente que "las penas con pan son menos", y la ansiedad se puede confundir con sensaciones interoceptivas de hambre. Se tendría que tener claro que el bajo NSE, la baja escolaridad, la mayor edad y el menor número de hijos son variables que también inciden en el fenómeno de la obe- 
sidad y que en la implementación de programas que tengan que ver con el tratamiento de esta problemática se deben de tomar en cuenta, pero no constituyen el único factor a considerar; variables psicológicas de personalidad, emocionales y conductuales complejizan el problema y, por tanto, su tratamiento.

Se debe advertir que, aunque otros estudios han reportado un menor control de impulsos en personas con obesidad (Mobbs, Crépin, Thiéry, Golay \& Van der Linden, 2010; Rand \& Stunkard, 1978), en este estudio la falta de control de impulsos no parece ser un predictor de la misma y, si bien existe la conducta de comer excesivo — pese a la presencia de motivación para bajar de peso en el grupo de mujeres obesas-, esta puede ser vista como una forma de fallo en dicho control (Bravo, Espinosa, Mancilla \& Tello, 2011; Rand \& Stunkard, 1978). No obstante, como función yoica no es predictiva.

Del conjunto de variables estudiadas, se destacan como predictores de mayor IMC una mayor insatisfacción con la imagen corporal, mayor motivación para bajar de peso, peores hábitos de salud, mayor edad, menor número hijos y NSE bajo. El modelo predictivo considerando estas siete variables explica casi la mitad de la varianza del IMC, pero presenta ligera colinealidad entre las variables. Estos resultados son congruentes con los de otros estudios que reportan la asociación de la insatisfacción con la imagen corporal y del NSE bajo con la obesidad (Arias et al., 2006; Sansone, et al., 2001). Si bien la pobreza de hábitos de salud, la mayor edad y el bajo NSE pueden entenderse como predictores de la obesidad, queda duda de cómo la insatisfacción con la imagen corporal se convierte en un predictor de este problema o si es el resultado de ella. Asimismo, no queda clara la forma en cómo la mayor motivación para bajar de peso dentro del grupo de obesos puede ser un predictor de su obesidad. ¿No debiera ser una menor motivación para bajar de peso la que llevase a la obesidad? Este modelo predictivo resulta confuso e invita a conocer un poco más a la población obesa, dado que sería interesante discernir cómo la motivación para bajar de peso no encuentra frutos en la solución del problema o de qué forma sus conductas de comer y hábitos de salud son una tranca difícil de vencer.

Es de destacar que la correlación entre el número de hijos y el IMC es directa: a mayor número de hijos se tiene mayor masa corporal. Precisamente el embarazo es un estresor para el cuerpo que genera aumento de peso y pérdida de la figura corporal, especialmente en casos de mujeres cuyos primeros embarazos fueron a edades adolescentes y cuentan con peor calidad de dieta por bajos recursos económicos (Baum \& Ruhm, 2009; Gunderson et al., 2009). No obstante, al parcializar el efecto de las otras variables en el modelo de regresión (edad, NSE, motivación para bajar de peso, imagen corporal y hábitos de salud), especialmente la edad, la correlación se hace inversa: a menor número de hijos se tiene mayor masa corporal. Esto refleja que en la presente muestra la obesidad se centra especialmente en un grupo de mujeres jóvenes sin hijos.

Se definieron tres modelos para predecir el IMC por análisis de senderos, manejando relaciones direccionales con mayor sentido causal que en el anterior modelo predictivo, además de contemplar relaciones intermedias y la correlación entre predictores exógenos.

El primer modelo indica que las mujeres con mayor IMC poseen insatisfacción con la imagen de su cuerpo y se hallan motivadas para bajar de peso. Quienes tienen mayor edad y menor NSE poseen mayor IMC. La primera vía es directa, siendo las mujeres con mayor masa corporal las que tienen una mayor motivación para bajar de peso. La segunda vía es indirecta, a través de la imagen corporal: el exceso de masa corporal genera insatisfacción con la imagen corporal y motiva a bajar de peso. Por otra parte, los hábitos de salud pronostican la motivación para bajar de peso y la imagen corporal: el modelo refleja que tener buenos hábitos de salud previene la insatisfacción con la imagen corporal y motiva a bajar de peso.

El segundo modelo indica que las mujeres con mayor masa corporal, malos hábitos de salud y que comen en exceso tienen mayor insatisfacción con su imagen corporal, las que, a su vez, tienen mayor motivación para bajar de peso. Asimismo, las mujeres de NSE más bajo, 
mayor edad y que reportan comer en exceso poseen mayor IMC. Se observa la presencia de peores hábitos de salud a menor NSE y mayor edad. Además, en este modelo aparecen como significativas las correlaciones del comer excesivo con peores hábitos de salud y mayor edad. Probablemente las mujeres de más edad, con estilos de vida sedentarios y metabolismos menos eficientes perciben fácilmente que ingieren un exceso de calorías, frente a las más jóvenes que son más activas y con un metabolismo más eficiente (Kola, Grossman \& Korbonits, 2008). Por último, en este modelo también los buenos hábitos de salud previenen la insatisfacción con la imagen corporal y motivan a bajar de peso.

En el tercer modelo la alteración afectiva se añade como un predictor con bastante peso del comer excesivo, como una forma de afrontamiento de malestar emocional, que podría denominarse autoindulgencia en el comer, y sensibiliza al malestar con la imagen corporal, siendo más frecuente la alteración afectiva a mayor edad. Esta autoindulgencia en el comer, matizada como un síntoma o forma de afrontar estados afectivos negativos, se desliga del discurso cotidiano estigmatizador en el que la obesidad sería consecuencia de un vicio (Friedman et al., 2005).

Estos modelos revelan la complejidad de las interacciones en tres factores que aquí se incluyen. No obstante, es preciso señalar que el estudio no es exhaustivo, por lo que no se agotan las variables que pueden estar presentes en diferentes grupos poblacionales que viven con esta problemática de salud. Es así que, respecto de las limitaciones del estudio, se debe advertir que, dadas las características no probabilísticas de la muestra empleada, la extrapolación de los resultados a una población femenina hispanoparlante semejante es limitada, por lo que los presentes resultados deben ser manejados como hipótesis. La muestra de este estudio también tiene un sesgo hacia un alto nivel de escolaridad, una escasa presencia de clase media-baja y un porcentaje alto de solteras, en comparación con la población general nuevoleonesa. También se desconoce la validez de los modelos para varones mexicanos, por lo que se recomienda incluirlos en futuras investigaciones, utilizando una muestra probabilística representativa de ambos sexos. Otra limitación es la naturaleza de autoreporte de los datos; ellos podrían diferir de resultados obtenidos por otros medios, como la observación o la entrevista.

En conclusión, tanto el sobrepeso como la obesidad se pueden volver una trampa difícil de franquear, ya que la alteración afectiva, en la que se incluyen aspectos de ansiedad, estrés y depresión, lleva a este grupo de mujeres jóvenes a comer en exceso, quizás como un intento de resolver el conflicto emocional. Eso incide en el aumento de peso y la insatisfacción con la imagen corporal, generada tras esa mayor grasa en el cuerpo, provoca mayor motivación para bajar de peso. A su vez, bajar de peso puede ser un factor de mayor ansiedad en la persona, ya que la restricción alimentaria que viene asociada con un régimen para bajar de peso genera mayor alteración alimentaria, en un ciclo que no tiene fin. No se trata solo de que las personas con mayor IMC coman más que otras personas, sino que no encuentran otra forma de manejar la ansiedad y el cuerpo puede volverse la mejor vía para manifestar un conflicto, siendo este el escenario de un drama que no halla desenlace. Aun así, se espera que el conflicto asociado con la obesidad disminuya con los años, quizás porque las características de personalidad varían en el curso de los años y por la influencia que el entorno tiene sobre las construcciones subjetivas (del cuerpo), las cuales varían conforme una persona adquiere una mayor maduración (Odgen, 2005). A mayor edad se espera asumir diferentes roles en la vida y el propio cuerpo deja de ser una preocupación central para las mujeres, pasando quizás a la preocupación por los hijos, el hogar y los padres ya ancianos, entre otras.

Desde este estudio y de otros afines en resultados (Baum \& Ruhm, 2009; Bravo et al., 2011; González \& Pompa, 2011), se puede entender que el fenómeno de la obesidad no solo se reduce a una mayor ingesta de calorías que las necesarias, sino que la presencia de alteraciones afectivas y el desarrollo de conductas inadecuadas de salud pueden ser obstáculos importantes, si no se abordan en un programa de reducción de peso. Es importante trabajar estos aspectos, a fin de que las preocupaciones actuales por la creciente obesidad de la población mexicana y 
las estrategias que se implementan desde el sector salud no queden en un esfuerzo infructuoso para paliar este complejo fenómeno. Con ello, la inclusión de profesionales de la psicología en los programas de reducción de peso se vuelve parte vital en el trabajo multidisciplinario, en donde se deben abordar variables afectivas, cognitivas y conductuales, además de incluir intervenciones psicoeducativas que permitan realizar la modificación de hábitos alimenticios y de ejercicios que posibiliten la reducción de peso.

Por otro lado, la psicología de la salud, que se ocupa del ámbito preventivo, podría promover formas más saludables de interacción con la comida y con el cuerpo, enseñando a padres e hijos que la función nutricia de la comida es básica y que esta debe ser sana y equilibrada, buscando la estabilidad del organismo.

\section{Referencias}

American Psychological Association (2002). Ethical principles of psychologists and code of conduct. American Psychologist, 57, 1060-1073. doi:10.1037/0003-066X.57.12.1060

Arias, F., Sánchez, S., Gorgojo, J. J., Almodóvar, F., Fernández, S. \& Llorente, F. (2006). Diferencias clínicas entre pacientes obesos mórbidos con y sin atracones. Actas Españolas de Psiquiatría, 34, 362-370. doi:10.1016/S15750922(06)71128-7

Baum, C. L. \& Ruhm, C. J. (2009). Age, socioeconomic status and obesity growth. Journal of Health Economics, 28, 635-648. doi:10.1016/j.jhealeco.2009.01.004

Bellak, L. \& Goldsmith, L. A. (1993). Metas amplias para la evaluación de las funciones del yo (J. A. Velásquez, Trad.). México DF, México: Manual Moderno.

Bravo, A., Espinosa, T., Mancilla, L. N. \& Tello, M. (2011). Rasgos de personalidad en pacientes con obesidad. Enseñanza e Investigación en Psicología, 16, 115-123. Extraído de http://redalyc.uaemex.mx/src/inicio/ArtPdfRed. jsp?iCve=29215963010

Bruch, H. (1973). Eating disorders: Obesity, anorexia nervosa and the person within. New York, NY: Basic Books.

Cabello, M. L. \& Zúñiga, J. G. (2007). Aspectos intrapersonales y familiares asociados a la obesidad: un análisis fenomenológico. Ciencia UANL, 10, 183-189. Extraído de http://eprints.uanl.mx/543/1/OBESIDAD.pdf

Chinchilla, A. (2005). Obesidad y psiquiatría. Barcelona, España: Masson.

Crosnoe, R. (2007). Gender, obesity, and education. Sociology of Education, 80, 241-260. doi:10.1177/0038040707080 00303

Flores, M., Carrión, C. \& Barquera, S. (2005). Sobrepeso materno y obesidad en escolares mexicanos. Encuesta Nacional de Nutrición, 1999. Salud Pública de México, 47, 447-450. doi:10.1590/S0036-36342005000600009

Friedman, K. E., Reichmann, S. K., Costanzo, P. R., Zelli, A., Ashmore, J. A. \& Musante, G. J. (2005). Weight stigmatization and ideological beliefs: Relation to psychological functioning in obese adults. Obesity Research, 13, 907-916. doi:10.1038/oby.2005.105

González, M. T. \& Pompa, E. G. (2011). Disminución de la ansiedad y el índice de masa corporal en niños con sobrepeso y obesidad, después de un tratamiento multidisciplinario. Ansiedad y Estrés, 17, 211-219.

Gunderson, E. P., Striegel-Moore, R., Schreiber, G., Hudes, M., Biro, S., Daniels, S. \& Crawford, P. B. (2009). Longitudinal study of growth and adiposity in parous compared with nulligravid adolescents. Archives of Pediatrics \& Adolescent Medicine, 163, 349-356. doi:10.1001/archpediatrics.2009.9

Hernández, B., Peterson, K., Sobol, A., Rivera, J., Sepúlveda, J. \& Lezana, M. A. (1996). Sobrepeso en mujeres de 12 a 49 años y niños menores de cinco años en México. Salud Pública de México, 38, 178-188. Extraído de http://bvs. insp.mx/rsp/_files/File/1996/num_3/38_3_sobrepeso.pdf

Hidalgo, C.G. \& Gottlieb, B. (2001). Comportamiento interpersonal de pacientes obesos y su relación con el éxito en un tratamiento para bajar de peso. Psykhe, 10(1), 59-68.

Himmelgreen, D. A. (2007). A fat: The anthropology of an obsession (2005). D. Kulick \& A. Meneley (Eds.). New York, NY: Jeremy P. Tarcher/Penguin [Book Reviews]. American Anthropologist, 109, 216-217. doi:10.1525/ aa.2007.109.1.216.1

Junien, C. \& Nathanielsz, P. (2007). Report on the IASO Stock Conference 2006: Early and lifelong environmental epigenomic programming of metabolic syndrome, obesity and type II diabetes. Obesity Reviews, 8, 487-502. doi:10.1111/j.1467-789X.2007.00371.x

Kilicarslan, A., Isildak, M., Sain Guven, G., Gul Oz, S., Durusu Tannover, M., Duman A. E. ... Sozen, T. (2006). Demographic, socioeconomic and educational aspects of obesity in an adult population. Journal of the National Medical Association, 98, 1313-1317. Extraído de http://www.ncbi.nlm.nih.gov/pmc/articles/PMC2569569/pdf/ jnma00195-0075.pdf

Kola, B., Grossman, A. \& Korbonits, M. (2008). The role of AMP-activated protein kinase in obesity. Frontiers of Hormone Research, 36, 198-211. doi:10.1159/000115366. Extraído de http://content.karger.com/ProdukteDB/ Katalogteile/isbn3_8055/_84/_29/fhr36_04.pdf

Lim, W., Thomas, K. S., Bardwell, W. A. \& Dimsdale, J. E. (2008). Which measures of obesity are related to depressive symptoms and in whom? Psychosomatics, 49, 23-28. doi:10.1176/appi.psy.49.1.23 
McCall, R. J. (1974). Group therapy with obese women of varying MMPI profiles. Journal of Clinical Psychology, 30, 466-470. doi:10.1002/1097-4679(197410)30:4<466::AID-JCLP2270300408>3.0.CO;2-6

México, Instituto Nacional de Salud Pública (2007). Encuesta Nacional de Salud y Nutrición 2006. Resultados por entidad federativa, Nuevo León. Cuernavaca, México: Autor, Secretaría de Salud. Extraído de http://www.insp. $\mathrm{mx} / \mathrm{ensanut/norte/NuevoLeon.pdf}$

México, Secretaría de Salud (2010). Acuerdo Nacional para la Salud Alimentaria. Estrategia contra el sobrepeso y la obesidad. México DF, México: Autor. Extraído de http://portal.salud.gob.mx/descargas/pdf/ANSA_acciones_ gobierno_federal.pdf

Meza, C. \& Moral, J. (en prensa). Validación de la versión en español del Cuestionario Sobreingesta Alimentaria (OQ) en una muestra de mujeres mexicanas. Revista Intercontinental de Psicología y Educación.

Mobbs, O., Crépin, C., Thiéry, C., Golay, A. \& Van der Linden, M. (2010). Obesity and the four facets of impulsivity. Patient Education and Counseling, 79, 372-377. doi:10.1016/j.pec.2010.03.003

Montero, J. C. (2001). Obesidad: una visión antropológica. Buenos Aires, Argentina: Universidad de Buenos Aires, Facultad de Medicina. Extraído de http://www.meducar.com.ar/cursos/files/ANTROPOLOGIA.pdf

Moral, J. (2006). Análisis factorial confirmatorio. En R. Landero \& M. T. González (Eds.), Estadística con SPSS y metodología de la investigación (pp. 445-528). México DF, México: Trillas.

Odgen, J. (2005). Psicología de la alimentación. Madrid, España: Morata.

O’Donnell, W. E. \& Warren, W. L. (2007). Cuestionario de sobreingesta alimentaria (OQ) (S. Olivare, Trad.). México DF, México: Manual Moderno.

Olaiz, G., Rivera, J., Shamah, T., Rojas, R., Villalpando, S., Hernández, M. \& Sepúlveda, J. (Eds.) (2006). Encuesta Nacional de Salud y Nutrición (ENSANUT 2006). Cuernavaca, México: Instituto Nacional de Salud Pública. Extraído de http://www.insp.mx/ensanut/ensanut2006.pdf

Organization for Economic Co-operation and Development (2008). OECD Health Data 2008 [Software Computacional y Archivo]. Paris, Francia: Autor/Institute for Research and Information in Health Economics. Extraído de http:// new.sourceoecd.org

Rand, C. S. \& Stunkard, A. J. (1978). Obesity and psychoanalysis. The American Journal of Psychiatry, 135, 547-551. Extraído de http://www.eric.ed.gov/PDFS/ED140133.pdf

Renman, C., Engström, I., Silfverdal, S. A. \& Åman, J. (1999). Mental health and psychosocial characteristics in adolescent obesity: A population-based case-control study. Acta Podiatrica, 88, 998-1003. doi:10.1111/j.16512227.1999.tb00196.x

Sansone, R. A., Wiederman, M. W. \& Monteith, D. (2001). Obesity, borderline personality symptomatology, and body image among women in a psychiatric outpatient setting. International Journal of Eating Disorders, $29,76-79$. doi:10.1002/1098-108X(200101)29:1<76::AID-EAT12>3.0.CO;2-O

Sociedad Mexicana de Psicología (2007). Código ético del psicólogo (4ª ed.). México DF, México: Trillas.

Stroe, J. G. (1995). Obesity and ego function (AT9606693). San Diego, CA: University of California.

Stunkard, A. J. (1959). Eating patterns and obesity. Psychiatric Quarterly, 33, 284-295. doi: 10.1007/BF01575455

Stunkard, A. J. (2000). Factores determinantes de la obesidad: opinión actual. En M. Peña \& J. Bacallao (Eds.), La obesidad en la pobreza: un nuevo reto para la salud pública (Publicación Científica $\mathrm{N}^{\circ}$ 576, pp. 27-32). Washington, DC: Organización Panamericana de la Salud. Extraído de http://books.google.cl/books?hl=es\&lr=\&id=_JIbrcSnb pcC\&oi=fnd\&pg=PA27\&dq=Factores+determinantes+de+la+obesidad+autor:stunkard\&ots=p14UnuEzgH\&sig=

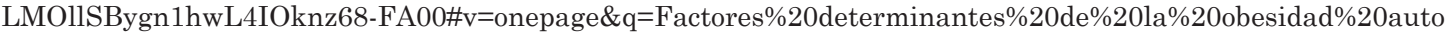
$\mathrm{r} \% 3$ Astunkard\&f $=$ false

Taylor, S. E. (2007). Psicología de la salud. México DF, México: McGraw Hill Interamericana.

Wadden, T. A. \& Lucas, R. A. (1980). MMPI as a predictor of weight loss. Psychological Reports, 46, 984-986. doi:10.2466/ pr0.1980.46.3.984

World Health Organization (2000). Obesity: Preventing and managing the global epidemic (Serie de Reportes Técnicos 894). Genève, Suiza: Autor. Extraído de http://whqlibdoc.who.int/trs/WHO_TRS_894.pdf

Fecha de recepción: Mayo de 2011.

Fecha de aceptación: Diciembre de 2012. 